\title{
ARTICLE
}

\section{A first-principles study on point defects in plutonium dioxide}

\author{
Hiroki Nakamura* and Masahiko Machida \\ CCSE, Japan Atomic Energy Agency, 178-4-4 Wakashiba, Kashiwa, Chiba, 277-0871, Japan
}

\begin{abstract}
Plutonium dioxide is one of the main components of nuclear fuels. In this paper, we evaluated formation energies of charged vacancies and site-interstitial atoms in plutonium dioxide based on density functional theory with spin-orbit coupling and strongly-correlated electron effect using LDA $+U$ method. We also estimated migration energies of oxygen defects. We found that oxygen vacancies with double positive charge and interstitials with double negative charge are the most stable and that the calculated migration energy of an oxygen vacancy is comparable with that of an oxygen interstitial.
\end{abstract}

\section{Keywords: plutonium dioxide; density-functional theory; point defect; vacancy; site-interstitial atom}

\section{Introduction}

Plutonium dioxide $\left(\mathrm{PuO}_{2}\right)$ is one of main components of mixed-oxide nuclear fuels, and therefore it is crucial to accumulate data of its physical properties. In particular, it is well known that vacancies and site-interstitial atoms (SIAs) strongly affects the physical properties of this material. Thus, it is important to investigate the effects of point defects such as vacancies and SIAs.

To investigate the effect of point defects, first-principles calculations are expected to play an important role, since it is difficult to measure their physical properties in detail. So far, a few calculations of formation exergies of charged point defects in actinide dioxides were reported $[1,2]$. The results of these papers implied that charge states of point defects are essential for estimation of their formation energies.

$\mathrm{PuO}_{2}$ is known to be a paramagnetic insulator. However, this material was sometimes calculated as an antiferromagnetic insulator using first-principles density functional theory (DFT), since non-relativistic DFT cannot predict an insulating state without antiferromagnetic order in the case of $\mathrm{PuO}_{2}$. In Ref. [3], we succeeded in obtaining the paramagnetic insulating state by considering spin-orbit coupling as relativistic effect and strong-correlation effect simultaneously. In this paper, we evaluate the formation energies of charged point defects in $\mathrm{PuO}_{2}$ and some of their migration energies, based on the paramagnetic insulating bulk state.

*Corresponding author. Email: nakamura.hiroki@jaea.go.jp

\section{Calculation methods}

\subsection{Ground-state calculation}

Plutonium dioxide has the fluorite crystal structure whose space group is $F m \overline{3} m$. This materials is well known as the van Vleck paramagnet. In all calculations, we adopt a $2 \times 2 \times 2$ supercell which contains $32 \mathrm{Pu}$ atoms and $64 \mathrm{O}$ atoms (Wyckoff positions $4 a$ and $8 c$, respectively) in the case of stoichiometric $\mathrm{PuO}_{2}$ as shown in Figure 1.

We employ VASP [4] as a DFT calculation package which supports the PAW method [5]. We adopt spin-orbit coupling as relativistic effect and DFT $+U$ method $(U=4 \mathrm{eV}$ and $J=0)$ as strongly-correlated electron effect. The value of $U$ was determined to fit the insulating gap size as shown in Ref. [3]. Recently, smaller value of $U(0.95 \mathrm{eV})$ was reported for pure plutonium using self-consistent constrained random-phase approximation (cRPA)[6]. However, the value of $U$ for oxides tends to be larger than that of pure metal, and therefore we can expect that the present value is reasonable. In fact, calculated $U$ of $\mathrm{UO}_{2}(5.7 \mathrm{eV})$ was

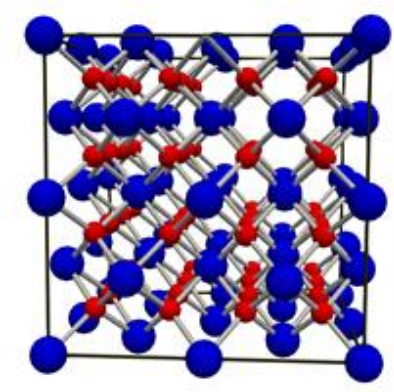

Figure 1. Crystal structure of $\mathrm{PuO}_{2}$. The $2 \times 2 \times 2$ supercell is shown here. Large and small spheres stand for $\mathrm{Pu}$ and $\mathrm{O}$ atoms, respectively. 

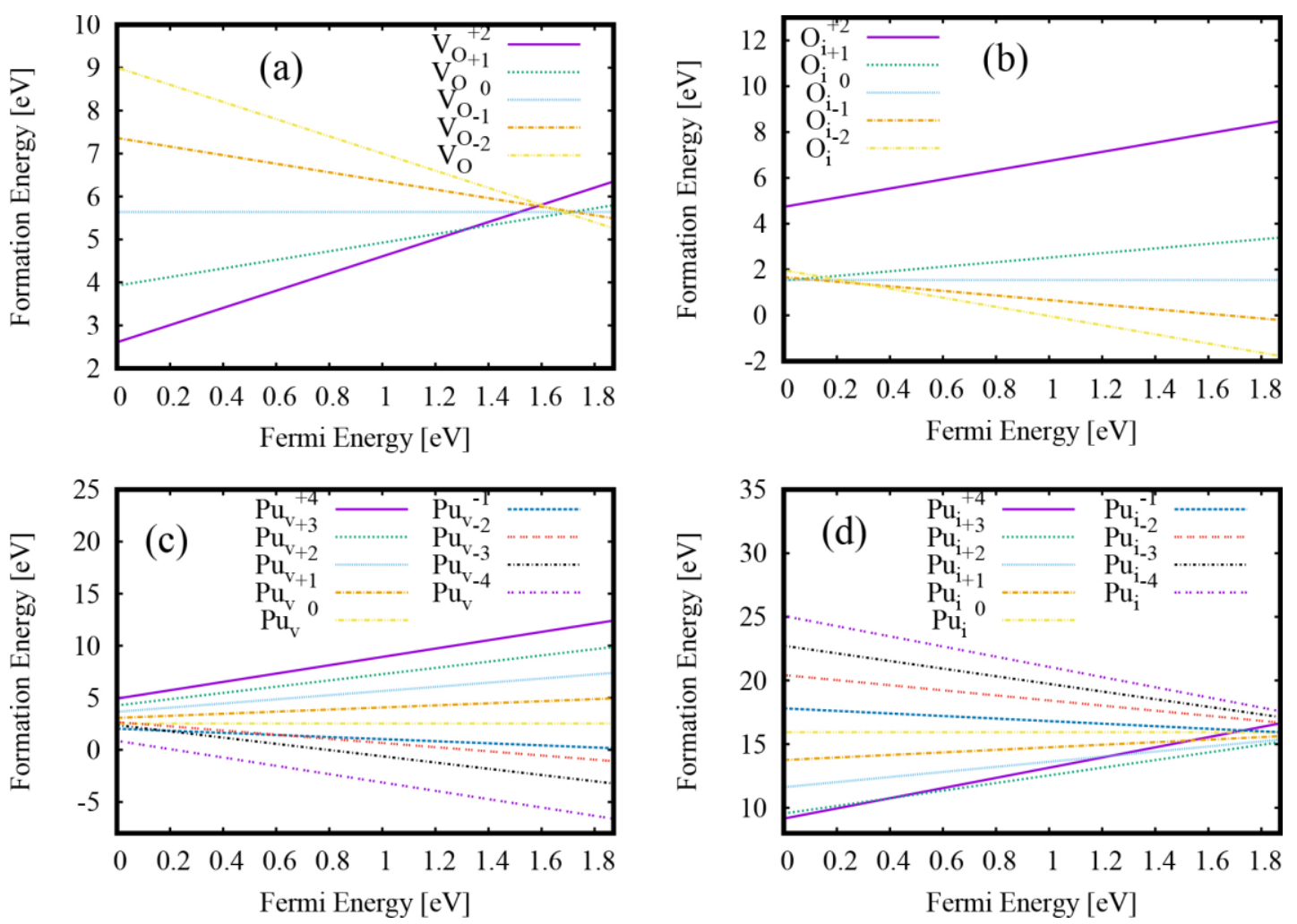

Figure 2. Formation energy of charged point defects as a function of the Fermi energy for oxygen vacancy (a), oxygen interstitial (b), plutonium vacancy (c) and plutonium interstitial (d).

reported to be larger than that of pure $\mathrm{U}$ metal $(0.8$ eV)[6,7].

The exchange-correlation energy we use is LDA [8], and the energy cutoff is set to $500 \mathrm{eV}$. The $k$-points are taken as $3 \times 3 \times 3$ through the paper. These setting values are similar to those in Ref. [3]. Therefore, the obtained state is also similar to that of the literature, i.e., the paramagnetic insulating state with an insulating gap (1.8 eV). As mentioned in Ref. [3], the calculated energy of the paramagnetic state becomes larger than that of magnetic state. However, we adopt the paramagnetic state as the ground state to evaluate defect formation energies, since correct defect structure cannot be obtained by distortion caused by artificial magnetic order. We optimize the lattice parameter and obtain $5.358 \AA$ which agrees with the experimental value 5.396 $\AA$ [9]. In all calculations, we fix the lattice parameter to the optimized value.

\subsection{Point defects}

The point defects focused in this paper are vacancies and interstitials of oxygen and plutonium atoms. The interstitial site we consider corresponds to the $4 b$ Wyckoff position, which is the center of an oxygen cube. We prepare one point defect in the calculation supercell. The charge of a defect is controlled by varying the number of electrons in the cell. The background charge is also changed to keep the calculation cell neutral. In the case of oxygen vacancy, we evaluate its formation energy through

$$
\begin{aligned}
E_{V_{o}} & =E\left(\mathrm{Pu}_{32} \mathrm{O}_{63}\right)-E\left(\mathrm{Pu}_{32} \mathrm{O}_{64}\right)+\frac{1}{2} E\left(\mathrm{O}_{2}\right) \\
& +q\left(E_{\mathrm{VBM}}+E_{\mathrm{F}}\right)
\end{aligned}
$$

where $E(X)$ is the calculated total energy of the supercell with composition $X$, which is obtained by optimizing atomic positions with fixed cell size. To obtain $E\left(\mathrm{O}_{2}\right)$, we prepare one oxygen molecule in the cell with the same size as that of $\mathrm{Pu}_{32} \mathrm{O}_{64}$. For the charged defects, the formation energies depend on the Fermi energy $E_{\mathrm{F}}$ which corresponds to the formation energy of an electron. In Eq. (1), $q$ denotes the charge of the point defect and $E_{\mathrm{VBM}}$ is the valence-band maximum energy. For interstitials, we can evaluate their formation energy similarly. Our calculation method basically follows those in Ref. [1,2]

Using calculated formation energies of charged point defects, we can evaluate the formation energies of neutral Frenkel and Schottky defects. In the case of a Frenkel defect, we consider a pair of $+q$ vacancy and $-q$ interstitial, and the formation energy of the Frenkel pair is obtained from the sum of the formation energies of the vacancy and interstitial. Thus, the formation energy of a neutral Frenkel defect no longer depends on the Fermi energy.

We also evaluate the migration energies of an oxygen vacancy and interstitial. To calculate the migration energy, we employ the nudged elastic band method [10] implemented in VASP with 5 intermediate images. 
Table 1. Formation energy of oxygen Frenkel defect.

\begin{tabular}{cccc}
\hline \multirow{2}{*}{$\begin{array}{c}\text { Vacancy } \\
\text { charge }\end{array}$} & SIA charge & \multicolumn{2}{c}{ Calculated formation } \\
& & energy (eV) \\
Present & Ref. [2] \\
work & \\
\hline+2 & -2 & 4.58 & 3.48 \\
+1 & -1 & 5.59 & 4.93 \\
0 & 0 & 7.18 & 6.05 \\
-1 & +1 & 8.88 & \\
-2 & +2 & 13.74 & \\
\hline
\end{tabular}

\section{Results and discussion}

\subsection{Charged defects}

Calculated formation energies of charged defects are shown in Figure 2. In the case of an oxygen vacancy, the +2-charged defect has the lowest formation energy up to $1.3 \mathrm{eV}$ of the Fermi energy. For an oxygen interstitial, the -2-charged state is the most stable in the wide range of the Fermi energy. These results are reasonable since an oxygen atom in $\mathrm{PuO}_{2}$ is expected to be a divalent anion. Similarly, in the case of plutonium which can be a tetravalent cation in this material, the -4-charged vacancy has the lowest formation energy. However, +3-charged interstitial plutonium atom has the lowest formation energy over $0.4 \mathrm{eV}$ of Fermi energy, while +4 -charged state is the more stable at low Fermi energy. This result is consistent with the fact that $\mathrm{PuO}_{2}$ tends to be hypo-stoichiometric, where some plutonium atoms can be trivalent cations.

\subsection{Frenkel and Schottky defects}

In Table 1, we summarize the formation energies of oxygen neutral Frenkel pairs for various charge states. The Frenkel pair of +2 -vacancy and -2 -interstitial is the most stable, whose formation energy is $4.58 \mathrm{eV}$. This result reflects that these defects are the most stable as mentioned in the previous subsection. To confirm the formation energy of an oxygen Frenkel defect, we also evaluate the total energy of an oxygen vacancy and interstitial in one supercell and obtain the similar formation energy $4.47 \mathrm{eV}$. Our result is slightly larger than the previously reported calculations using

(a)

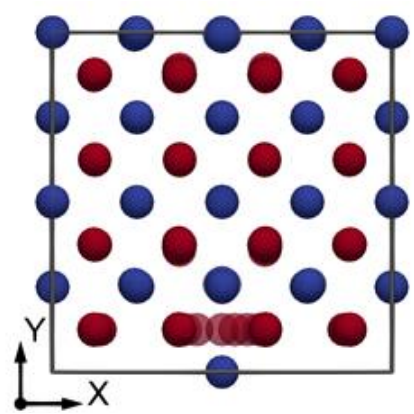

Table 2. Formation energies of various neutral defects. $\mathbf{V}_{\mathbf{X}}^{\boldsymbol{q}}$ and $\mathbf{I}_{\mathbf{X}}^{q}$ denotes vacancy and interstitial of element $\mathrm{X}$ with charge $q$, respectively.

\begin{tabular}{ccc}
\hline Type of neutral defect & Charge states & $\begin{array}{c}\text { Calculated } \\
\text { formation } \\
\text { energy }(\mathrm{eV})\end{array}$ \\
\hline $\mathrm{Pu}$ Frenkel defect & $\mathrm{V}_{\mathrm{Pu}}^{-4}+\mathrm{I}_{\mathrm{Pu}}^{+4}$ & 10.02 \\
$\mathrm{PuO}_{2}$ Schottky defect & $\mathrm{V}_{\mathrm{Pu}}^{-4}+2 \mathrm{~V}_{\mathrm{O}}^{+2}$ & 6.09 \\
$\mathrm{PuO}_{2}$ interstitial & $\mathrm{I}_{\mathrm{Pu}}^{+4}+2 \mathrm{I}_{\mathrm{O}}^{-2}$ & 13.09 \\
\hline
\end{tabular}

antiferromagnetic ground state [2] as shown in Table 1.

We also evaluate formation energies of other neutral defects as summarized in Table 2 . The formation energies tabulated in this table are larger than that of oxygen Frenkel pair in Table 1.

\subsection{Migration energy}

We evaluate migration energies of +2 -charged oxygen vacancy and -2 -charged interstitial. We assume that an oxygen vacancy moves to the nearest oxygen-site as shown in Figure 3a. In the case of oxygen interstitial migration, the interstitial atom pushes out the nearest oxygen, and the pushed atom moves to another interstitial site (Figure 3b). If the interstitial atom moves directly to another interstitial site, the migration energy becomes much larger than that of the former migration path. The calculated migration energies are $1.11 \mathrm{eV}$ and $1.18 \mathrm{eV}$ for an oxygen vacancy and interstitial, respectively. These results imply that the mobility of interstitials are not so different from that of vacancies.

\subsection{Comparison with literature}

The measured formation energy of an oxygen Frenkel pair and migration energies of an oxygen vacancy and interstitial were summarized in Ref. [11]. The calculated formation energy of the oxygen Frenkel defect is slightly larger than observed values $2.72-2.92 \mathrm{eV}$. The reported migration energies are $0.48 \mathrm{eV}$ and $1.58 \mathrm{eV}$ for an oxygen vacancy and interstitial, respectively. The calculated value for interstitial migration are slightly smaller than the measurement, while the migration (b)

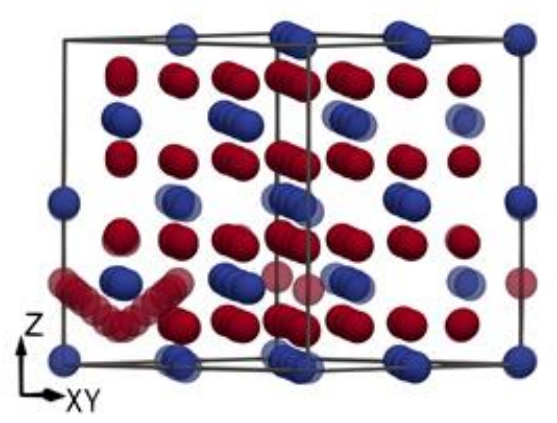

Figure 3. Migration paths for oxygen vacancy (a) and oxygen interstitial (b). Blue and red spheres denote $\mathrm{Pu}$ and $\mathrm{O}$ atoms, respectively. 
energy of a vacancy is larger than those of experiments. We can evaluate the activation energy of oxygen self-diffusion through the relation

$$
E_{\mathrm{A}}=\frac{E_{\text {Frenkel }}}{2}+E_{\text {mig }}
$$

where $E_{\text {Frenkel }}$ and $E_{\text {mig }}$ are the Frenkel energy and migration energy, respectively. The estimated activation energy from the calculated values is $3.4 \mathrm{eV}$, which is larger than the reported value $1.83-1.93 \mathrm{eV} \mathrm{[11].} \mathrm{We}$ should note that recent molecular dynamics calculations estimate much larger activation energy $(\sim 6 \mathrm{eV})[12]$.

The formation energies and migration energies of oxygen defects in $\mathrm{PuO}_{2}$ have not often been measured so far. On the other hand, the measured formation energy of Frenkel pair in $\mathrm{PuO}_{2}$ was reported to be smaller than that of $\mathrm{UO}_{2}(\sim 4 \mathrm{eV})[11]$, while the calculated values for $\mathrm{PuO}_{2}$ and $\mathrm{UO}_{2}$ are similar $\left(4.58 \mathrm{eV}\right.$ for $\mathrm{PuO}_{2}$ and $4.8 \mathrm{eV}$ for $\mathrm{UO}_{2}$ [1]). Thus, it is possible that some specific mechanism affects the measurement of the formation energy in $\mathrm{PuO}_{2}$. In the present situation, we might mention that our calculation results almost agree with the experimental results.

In our results, -2-charged interstitial oxygen is more stable in the wide range of Fermi energy. On the other hand, Prodan et al. pointed out that interstitial oxygen is -1-charged [13]. Even in our calculations, -1-charged interstitial is more stable than -2-charged one at low Fermi energy, as shown in Figure 2(b), and therefore our results do not necessarily conflict with them. The charges of defects are sensitive to their circumstance, such as oxygen partial pressure. Thus, comprehensive analyses would be required to determine the exact charge states.

\section{Conclusions}

We evaluated the formation energies of charged point defects in plutonium dioxide using density functional theory. In the calculations, we employed $\mathrm{LDA}+U$ with spin-orbit coupling and then our calculations were based on the paramagnetic insulating ground state of $\mathrm{PuO}_{2}$. We also estimated the formation energies of neutral Frenkel and Schottky defects. The migration energies of oxygen vacancy and interstitial were calculated, and we found that these values are similar. Though our calculation results slightly deviate from experimental values, they are still comparable with experiments.

\section{Acknowledgements}

The authors wish to thank M. Kato and M. Watanabe for illuminating discussion. The calculations were performed in the supercomputer ICE-X operated by CCSE, JAEA. We would like to thank all staff members of CCSE. This work was supported by JSPS KAKENHI Grant Number 16K06964.

\section{References}

[1] J.-P. Crocombette, D. Torumba and A. Chartier, Charge states of point defects in uranium oxide calculated with a local hybrid functional for correlated electrons, Phys. Rev. B. 83 (2011), p. 184107

[2] Y. Lu, Y. Yang and P. Zhang, Charge states of point defects in plutonium oxide: A first-principles study, J. Alloys Compd. 649 (2015), pp. 544-552.

[3] H. Nakamura, M. Machida and M. Kato, Effects of spin-orbit coupling and strong correlation on the paramagnetic insulating state in plutonium dioxides, Phys. Rev. B. 82 (2010), p. 155131.

[4] G. Kresse and J. Furthmüller, Efficient iterative schemes for $a b$ initio total-energy calculations using a plane-wave basis set, Phys. Rev. B. 54 (1996), pp. 11169-11186.

[5] P.E. Blöchl, Projector augmented-wave method, Phys. Rev. B. 50 (1994), pp. 17953-17979.

[6] B. Amadon, First-principles DFT+DMFT calculations of structural properties of actinides: Role of Hund's exchange, spin-orbit coupling, and crystal structure, Phys. Rev. B. 94 (2016), pp. 1-14.

[7] B. Amadon, T. Applencourt and F. Bruneval, Screened Coulomb interaction calculations: cRPA implementation and applications to dynamical screening and self-consistency in uranium dioxide and cerium, Phys. Rev. B. 89 (2014), p.125110.

[8] S.H. Vosko, L. Wilk and M. Nusair, Accurate spin-dependent electron liquid correlation energies for local spin density calculations: a critical analysis, Can. J. Phys. 58 (1980), pp. 1200-1211.

[9] D.L. Clark, S.S. Hecker, G.D. Jarvinen and M.P. Neu, Chapter 7 Plutonium, in: L.R. Morss, N.M. Edelstein, J. Fuger (Eds.), The Chemistry of the Actinide and Transactinide Elements, 3rd ed., Springer Netherlands, Dordrecht (2006), pp. 813-1264.

[10]G. Mills, H. Jónsson and G.K. Schenter, Reversible work transition state theory: application to dissociative adsorption of hydrogen, Surf. Sci. 324 (1995), pp. 305-337.

[11]G.E. Murch and C.R.A. Catlow, Oxygen diffusion in $\mathrm{UO}_{2}, \mathrm{ThO}_{2}$ and $\mathrm{PuO}_{2}$. A review, J. Chem. Soc. Faraday Trans. 2.83 (1987), p. 1157.

[12]V. Saltas, A. Chroneos, M.W.D. Cooper, M.E. Fitzpatrick and F. Vallianatos, Investigation of oxygen self-diffusion in $\mathrm{PuO}_{2}$ by combining molecular dynamics with thermodynamic calculations, RSC Adv. 6 (2016), pp. 103641-103649.

[13]I.D. Prodan, G.E. Scuseria, J.A. Sordo, K.N. Kudin and R.L. Martin, Lattice defects and magnetic ordering in plutonium oxides: a hybrid density-functional-theory study of strongly correlated materials, J Chem Phys. 123 (2005), p. 14703. 